Jean-Marie Ruysschaert

\title{
Rôle de motifs protéiques amphiphiles dans les processus de reconnaissance
}

La reconnaissance d'un récepteur par son ligand ou du site actif d'une enzyme par son substrat implique une très grande spécificité. Cependant, un certain nombre d'observations contredisent ce modèle : des apolipoprotéines de séquences très différentes manifestent des activités biologiques très proches; le nombre de séquences peptidiques responsables du ciblage des protéines mitochondriales dépasse sans doute le nombre de récepteurs disponibles sur la membrane mitochondriale; la calmoduline s'associe à des protéines très différentes, et module leur activité biologique, etc. L'examen des séquences impliquées dans ces processus de reconnaissance montre que celles-ci adoptent une structure hélicoïdale amphiphile ou amphipathique au sein de laquelle les acides aminés hydrophiles et hydrophobes se répartissent en deux domaines distincts. C'est la distribution des acides aminés hydrophobes et hydrophiles plutôt que leur séquence qui détermine une reconnaissance à large spectre.

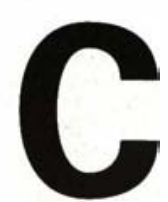

lassiquement, le processus de reconnaissance entre un récepteur et son ligand, le site actif d'une enzyme et son substrat, implique un mécanisme d'une grande spécificité. Cependant, un tel mécanisme rend difficilement compte d'un certain nombre d'observations, et notamment du fait qu'une séquence peptidique ou protéique peut reconnaître plusieurs partenaires différents. Qu'est-ce qui permet une telle souplesse dans la reconnaissance moléculaire? C'est ce que nous vou- drions faire comprendre par quelques exemples significatifs ; la liste n'est évidemment pas exhaustive.

\section{Interchangeabilité de domaines impliqués dans la fusion virus-cellule hôte}

De façon très schématique, le mécanisme d'infection d'une cellule par un rétrovirus procède en plusieurs étapes : (1) fixation du virus sur la cellule cible ; $(2)$ fusion de l'enveloppe virale et de la membrane plas- 
mique de la cellule hôte et injection du matériel génétique dans la cellule ; (3) réplication du matériel viral. La première étape est très spécifique et requiert la présence d'un récepteur sur la membrane plasmique. La seconde étape procède par un mécanisme plus général mais qui reste mal compris, du moins en termes moléculaires. Dans le cas du VIH (virus de l'immunodéficience humaine) ( $\mathfrak{i g u -}$ re 1), la reconnaissance entre une protéine virale gp120 et le récepteur

\section{RÉFÉRENCES}

1. Eiden L, Lifson, J. HIV interactions with $\mathrm{CD}_{4}$, a continuum of conformations and consequences. Immunol Today $1992 ; 13$ : 201-6.

2. Kowalski M, Potz J, Basiripour L, Dorfman T, Goh W, Terwilliger E, Dayton A, Rosen L, Haseltine W, Sodroski J. Functional regions of the envelope glycoprotein of HIV type-I. Science 1987 ; 237 : 1351-5.

3. Vonèche $V$, Portetelle $D$, Kettmann $R$, Willems L, Paoletti E, Ruysschaert JM, Burny A, Brasseur R. Fusogenic segments of $B L V$ and SIV are interchangeable and mediate fusion via oblique insertion in the lipid bilayer of their target cells. Proc Natl Acad Sci USA $1992 ; 89$ : 3810-4.

4. Brasseur R, Vandenbranden M, Cornet B, Burny A, Ruysschaert JM. Orientation into the lipid bilayer of an asymmetric amphipathic helical peptide located at the N-terminus of viral fusion proteins. Biochim Biophys Acta $1990 ; 1029$ : 267-73.

5. Horth M, Lambrecht MC, Lay Khim MC, Bex F, Thiriart C, Ruysschaert JM, Burny A, Brasseur $\mathbf{R}$. Theoretical and functional analysis of the SIV fusion peptide. EMBO J 1991 ; $10: 2747-55$.

6. Owens RJ, Anantharamaiah GM, Kahlon JB, Srinivas RV, Compans RW, Segrest JP. Apolipoprotein A-I and its amphipathic helix peptide analogues inhibit phipathic helix peptide analogues inhibit syncytium formation. J Clin Invest $1990 ; 86$ : $1142-50$.

7. Martin I, Dubois MC, Saermark T, Ruysschaert JM. Apolipoprotein AI interaction with the N-terminal domain of SIV gp32 and HIV gp41. Biochem Biophys Res Commun $1992 ; 186: 95-101$.

8. Vonèche $V$, Callebaut $I$, Kettmann $R$, Brasseur R, Burny A, Portetelle D. The 19 27 amino acid segment of gp51 adopts an amphiphilic structure and plays a key role
in the fusion events induced by bovine leu-

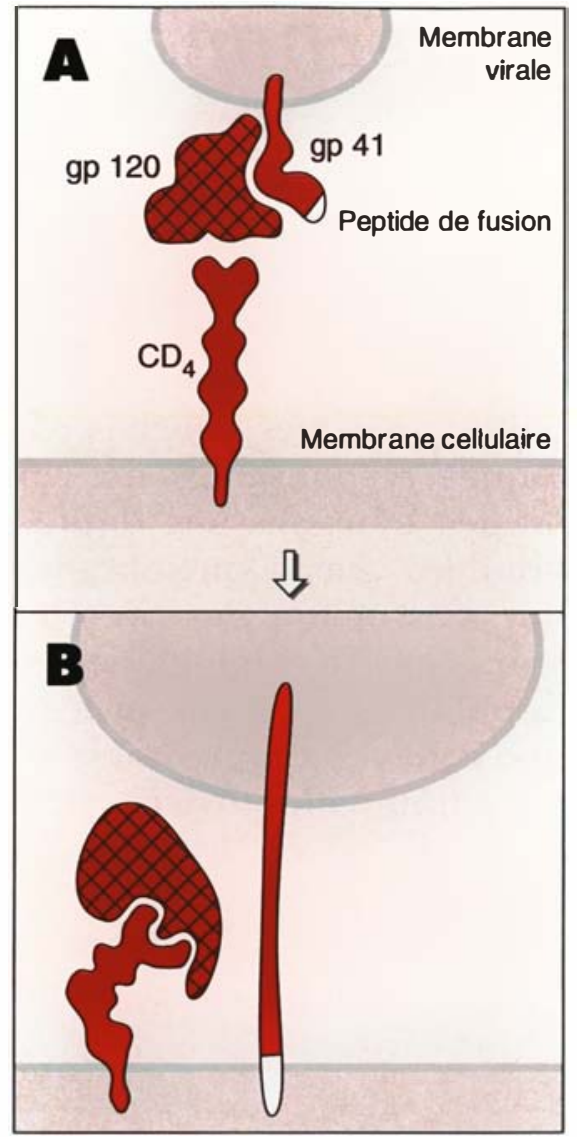

Figure 1. Représentation schématique du mode d'interaction d'un rétrovirus (VIH) avec sa cellule cible. A. La première étape, très spécifique, est l'interaction d'une protéine virale (gp120) avec un récepteur sur la membrane plasmique (CD4). B. La deuxième étape suit un changement conformationnel des protéines virales : les membranes virale et cellulaire se rapprochent et le peptide $\mathbf{N}$ terminal hydrophobe de la gp41 pénètre dans la bicouche lipidique de la cellule hôte, ce qui déstabilise la membrane et permet le mélange des phases lipidiques et aqueuses. cellulaire $\mathrm{CD}_{4}$ induirait une modification de la structure des protéines virales [1]. Ce changement conformationnel aurait un double effet : d'une part, le rapprochement des membranes virales et cellulaires et, d'autre part, la pénétration du peptide N-terminal hydrophobe de la gp4l dans la bicouche lipidique de la cellule hôte avec pour conséquence la déstabilisation de la membrane et le mélange des phases lipidiques et aqueuses. La participation de ce peptide $\mathrm{N}$-terminal au processus de fusion a été démontrée par des expériences de mutagenèse dirigée [2], notamment dans le cas du VIH.

La caractéristique principale du domaine N-terminal de la protéine de fusion des rétrovirus est son hydrophobicité. Cependant, des travaux récents suggèrent que ce n'est sans doute pas le seul paramètre essentiel. En effet, le gène codant pour la protéine de fusion de BLV (bovine leukemia virus) a été modifié de telle façon que la protéine de fusion du BLV porte le domaine fusogène du SIV (simian immunodeficiency virus). Des lymphocytes ont été infectés par les vaccins recombinants et la formation de syncytia (cellules polynucléées) a été utilisée comme critère d'activité fusogène. La formation de cellules polynucléées résulte essentiellement de la fusion des cellules infectées portant des protéines virales fusogènes à leur surface et de cellules non infectées. La substitution du domaine N-terminal n'affecte pas le processus de formation de syncytiums [3]. Fait intéressant, les séquences des extrémités $\mathrm{NH}_{2}$-terminales du SIV (Gly-Val-Phe-Val-Leu-GlyPhe-Leu-Gly-Phe-Leu-Ala) et du BLV (Ser-Pro-Val-Ala-Ala-Leu-Thr-Leu-GlyLeu-Ala-Leu) sont différentes mais la distribution des acides aminés hydrophobes et hydrophiles à la surface d'une structure hélicoïdale est similaire [4]. Ce rassemblement des acides aminés hydrophobes et hydrophiles sur la surface de l'hélice confère une double affinité pour un environnement hydrophile et hydrophobe. On parle dans ce cas d'hélice amphiphile. Il a été suggéré que ce type de distribution jouerait un rôle déterminant dans la déstabilisation de la bicouche lipidique qui précède le mélange des phases aqueuse et lipidique du virus et de la cellule ; il caractérise le domaine 
fusogène $\mathrm{N}$-terminal de plusieurs virus $[4,5]$.

Cette constatation a stimulé la mise en œuvre d'une recherche à visée thérapeutique. En effet, l'association d'un peptide amphiphile au peptide fusogène devrait masquer le domaine hydrophobe ou hydrophile, altérer le motif amphiphile et donc l'activité fusogène. Ainsi, l'Apo AI [6] bloque l'activité fusogène du virus VIH, ou plus exactement sa capacité d'induire la formation de syncytiums. Cette protéine est, comme l'indique la figure $2 A$, constituée d'une succession de structures hélicoïdales amphipathiques susceptibles d'interagir avec l'extrémité $\mathrm{NH}_{2}$-terminale du VIH ou du SIV [7].

Ce type d'approche, fondée sur un spectre de reconnaissance peptidepeptide relativement peu spécifique, laisse augurer d'une activité antivirale à large spectre, c'est-à-dire contre une série de virus d'une même famille. Dans la structure virale, ce peptide fusogène est d'ailleurs vraisemblablement protégé de l'environnement aqueux par une structure peptidique complémentaire, associée à la protéine de fusion ou à la protéine de reconnaissance. Cela a été bien illustré dans le cas du BLV [8]. En effet, une modification de la distribution des acides aminés hydrophobes et hydrophiles dans le peptide protégeant le peptide $\mathrm{N}$-terminal modifie les propriétés fusogènes [8]. Le rôle de peptides antiviraux potentiels serait de rompre de telles associations.

\section{Les apolipoprotéines \\ responsables du transport du cholestérol et des lipides : des séquences très différentes caractérisées par la répétition $d^{\prime} u n$ même motif amphiphile}

Plusieurs apolipoprotéines (Apo A-I, Apo A-IV, Apo-E) $[9,10]$ possèdent la propriété de s'associer aux phospholipides et de permettre la formation de structures lipidiques discoïdales dont le diamètre est d'environ $100 \AA$ et l'épaisseur celle d'une bicouche lipidique. C'est sous cette forme que les HDL (high density lipoproteins) sont synthétisées par le foie. Ces disques lipidiques (figure 3) sont ca$m / s n^{\circ} 2$, vol. 11, férira 95 pables, dans un certain nombre de cas, d'extraire le cholestérol des cellules [11] dans lesquelles il s'est accumulé et de le transformer, via l'intervention d'une enzyme spécifique, la lécithine cholestérol acyltransférase, en ester de cholestérol. L'ester de cholestérol migre vers le centre de la particule et lui confère une forme sphérique. C'est sous cette forme, caractéristique des HDL circulant dans le plasma, que le cholestérol est éliminé via le foie.

La formation de disques lipidiques pose cependant un problème de stabilité. En effet, comment les chaînes acylées des phospholipides vont-elles échapper à l'environnement aqueux? La réponse se trouve dans un examen plus approfondi de la séquence de différentes apolipoprotéines. $\mathrm{Si}$ ces séquences sont différentes, elles se caractérisent par une succession de do-

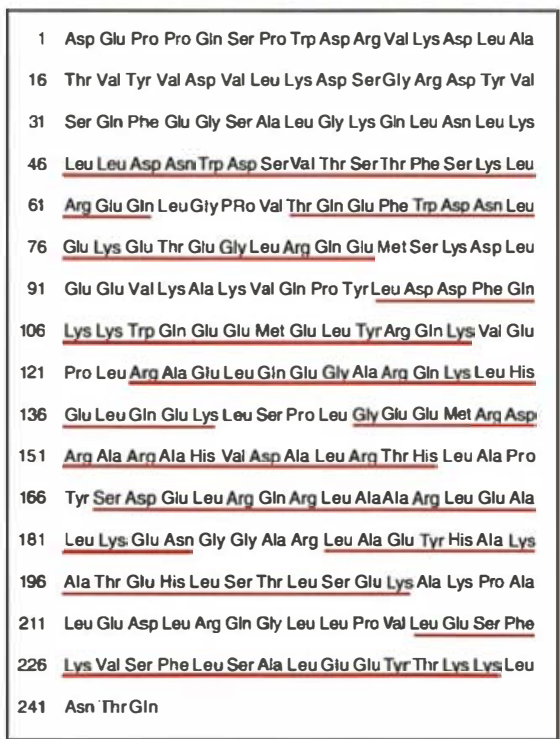

Figure 2. Apolipoprotéine. A. Séquence de l'apolipoprotéine A-I, les huit domaines devant former une hélice amphipathique sont soulignés. B. Représentation des hélices amphipathiques. Une façon de visualiser plus aisément la distribution des acides aminés est de projeter l'hélice sur le plan de la feuille et de disposer un acide aminé tous les $100^{\circ}$, (1) de l'Apo A-I (Ala 124 Leu 141) ; (2) du peptide synthétique [15].

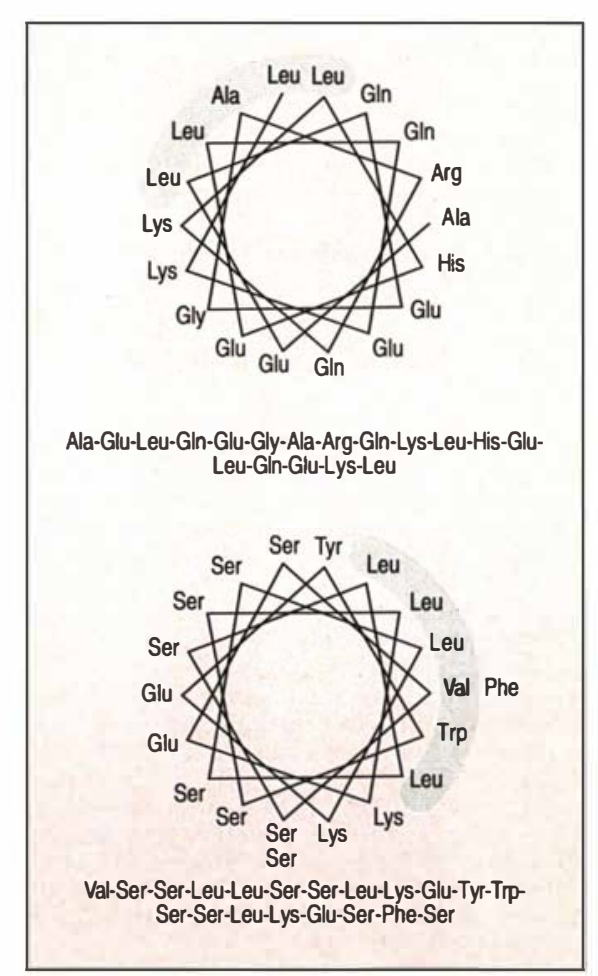

maines peptidiques susceptibles de s'organiser en hélices amphiphiles acides aminés dont généralement un résidu proline. Chaque séquence amphipathique est constituée d'une vingtaine d'acides aminés. La distribution des acides aminés sur l'hélice amphipathique se caractérise par un domaine hydrophobe moins étendu que le domaine hydrophile (figure $2 B$ ) [12]. La face hydrophobe de chacune de ces hélices s'associerait à la chaîne acylée des phospholipides alors que le domaine hydrophile s'orienterait vers la phase aqueuse [12]. Ce modèle est en accord avec le fait expérimental en ce qui concerne le nombre d'apolipoprotéines par particule, la dimension du disque, et l'orientation des hélices amphipathiques dans le complexe [12-14].

Ce type d'organisation pourrait per(figure $2 A$ ) et séparés par quelques 


\section{RÉFÉRENCES}

9. Gong EL, Nichols AV, Weisgraber KH, Forte TM, Shore VG, Blanche PJ. Discoidal complexes containing Apo-E and their transformation by LCAT. Biochim Biophys Acta $1989 ; 1006: 317-28$.

10. Matz CE, Jonas A. Micellar complexes of human Apo A-I with phosphatidylcholine and cholesterol prepared from cholate-lipid dispersions. J Biol Chem 1982 ; 257 : 4535-40.

11. Steinmetz A, Barbaras R, Ghalim N, Clavey V, Fruchart TC, Ailhaud G. Human apolipoprotein A-IV binds to Apo A-I, Apo A-II receptor sites and promotes cholesterol efflux from adipocyte cells. J Biol Chem 1990 ; 265: 7859-63.

12. Brasseur R, Lins L, Vanloo B, Ruysschaert IM, Rosseneu M. Molecular modeling of the amphipathic helices of the plasma apolipoproteins. Proteins: Simucture, Function and Genetics 1992 ; 13 : 246-57.

13. Hefele-Wald E, Goormaghtigh E, De Meutter J, Ruysschaert JM, Jonas A. Investigation of the lipid domains and apolipoprotein orientation in reconstituted high density lipoproteins by fluorescence and IR methods. J Biol Chem 1990 ; 265 : 20044-50.

14. Goormaghtigh E, Cabiaux V, Ruysschaert JM. Secondary structure and dosage of soluble and membrane proteins by attenuated total reflection Fourier-transform in frared spectroscopy on hydrated films. EurJ Biochem $1990 ; 193$ : 409-20.

15. Pownall HJ, Hu A, Gotto AM, Albers JJ, Sparrow JT. Activation of lecithin cholesterol acyltransferase by a synthetic model lipid-associating peptide. Proc Natl Acad Sci USA $1980 ; 77$ : 3154-8.

16. Verner K, Schatz G. Protein translocations across membranes. Science 1988 ; 241 : 1307-12.

17. O'Neil KT, De Grado WF. How calmodulin binds its targets : sequence independent recognition of amphiphilic $\alpha$-helices. mettre d'expliquer pourquoi des protéines de séquences différentes conservent une activité biologique commune : à savoir la formation de disques lipidiques correspondant aux HDL natives et l'activation d'une enzyme spécifique, la lécithine cholestérol acyltransférase.

Au niveau de la formation de disques, ce serait la répartition des domaines hydrophiles et hydrophobes plutôt que la succession des acides aminés qui permettrait une reconnaissance à large spectre de la phase lipidique. Quant à l'activation de la lécithine cholestérol acyltransférase, comment une enzyme possédant à la fois une activité de phospholipase (clivage d'une chaîne acylée) et d'acyltransférase (transfert d'une chaîne acylée au cholestérol) peut-elle être activée par des apolipoprotéines de séquences différentes ? Il est vraisemblable, mais cela ne reste qu'une hypothèse, que ce sont des interactions entre motifs amphiphiles associés à l'apolipoprotéine et l'enzyme qui permettent une première reconnaissance. Dans une deuxième étape, l'enzyme, associée aux disques lipidiques, pourrait procéder à l'interface lipideeau à l'estérification du cholestérol.

Il est intéressant de mentionner que des peptides synthétiques d'une vingtaine d'acides aminés possèdent la propriété de former des disques lipidiques et d'activer la lécithine cholestérol acyltransférase [15], quoique cette activité reste notablement inférieure à celle observée pour l'apolipoprotéine entière. Cependant, leur séquence ne correspond à aucune des séquences rencontrées dans les apolipoprotéines. Si les séquences sont différentes, la distribution des acides aminés hydrophobes et hydrophiles est néanmoins conservée (figure 2B). Certaines éventualités thérapeutiques, visant notamment à une élévation de la concentration de HDL capables d'éliminer le cholestérol accumulé dans les cellules, peuvent être envisagées, supposant, évidemment, que l'on dispose de quantités importantes d'apolipoprotéines purifiées. Un certain nombre de groupes se sont engagés dans cette voie en produisant des protéines par ingénierie génétique. Le remplacement de l'apolipoprotéine par un peptide synthétique simplifierait grandement cette approche.

\section{Un très grand nombre de peptides signaux, mais un nombre limité de récepteurs}

De courtes séquences peptidiques appelées peptide signal sont responsables de l'association de protéines, synthétisées ou en voie de synthèse, aux membranes du réticulum endoplasmique, des mitochondries ou des chloroplastes. Ces séquences sont diverses dans leur composition et en tout cas beaucoup plus nombreuses que les récepteurs membranaires déjà identifiés. Toutefois, l'examen des séquences fait apparaître, au-delà de leur diversité, une distribution des domaines hydrophobes et hydrophiles caractéristique. Ainsi, l'extrémité $\mathbf{N H}_{2}$ du peptide signal spécifique du réticu-

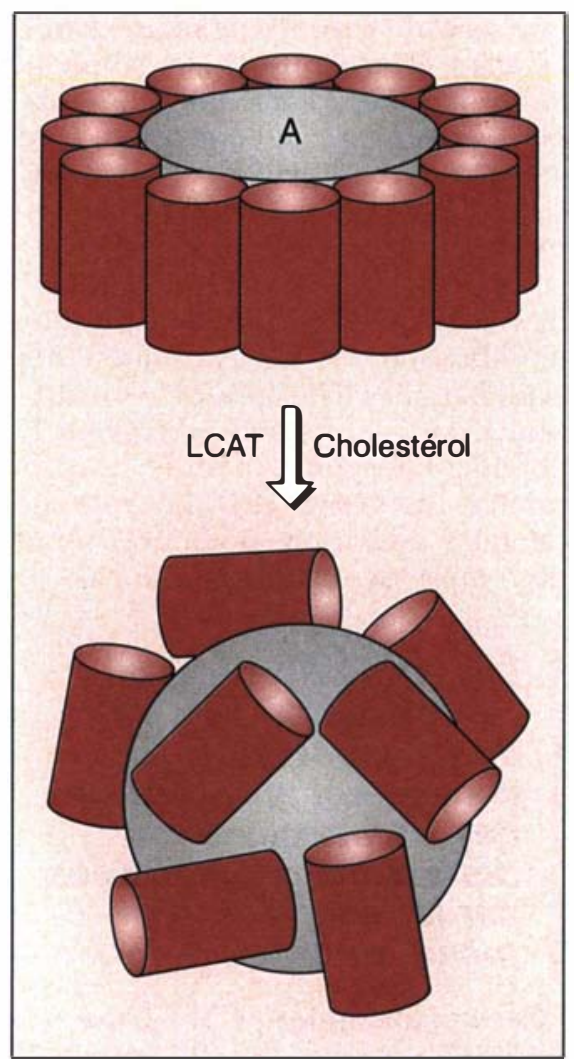

Figure 3. Représentation schématique de l'organisation des hélices amphipathiques des apolipoprotéines autour du cour lipidique (A) constitué par une bicouche lipidique (dimyristoy/phosphatidylcholine) avant et après transformation par action de la LCAT (lecithin cholesterol acyltransferase). 


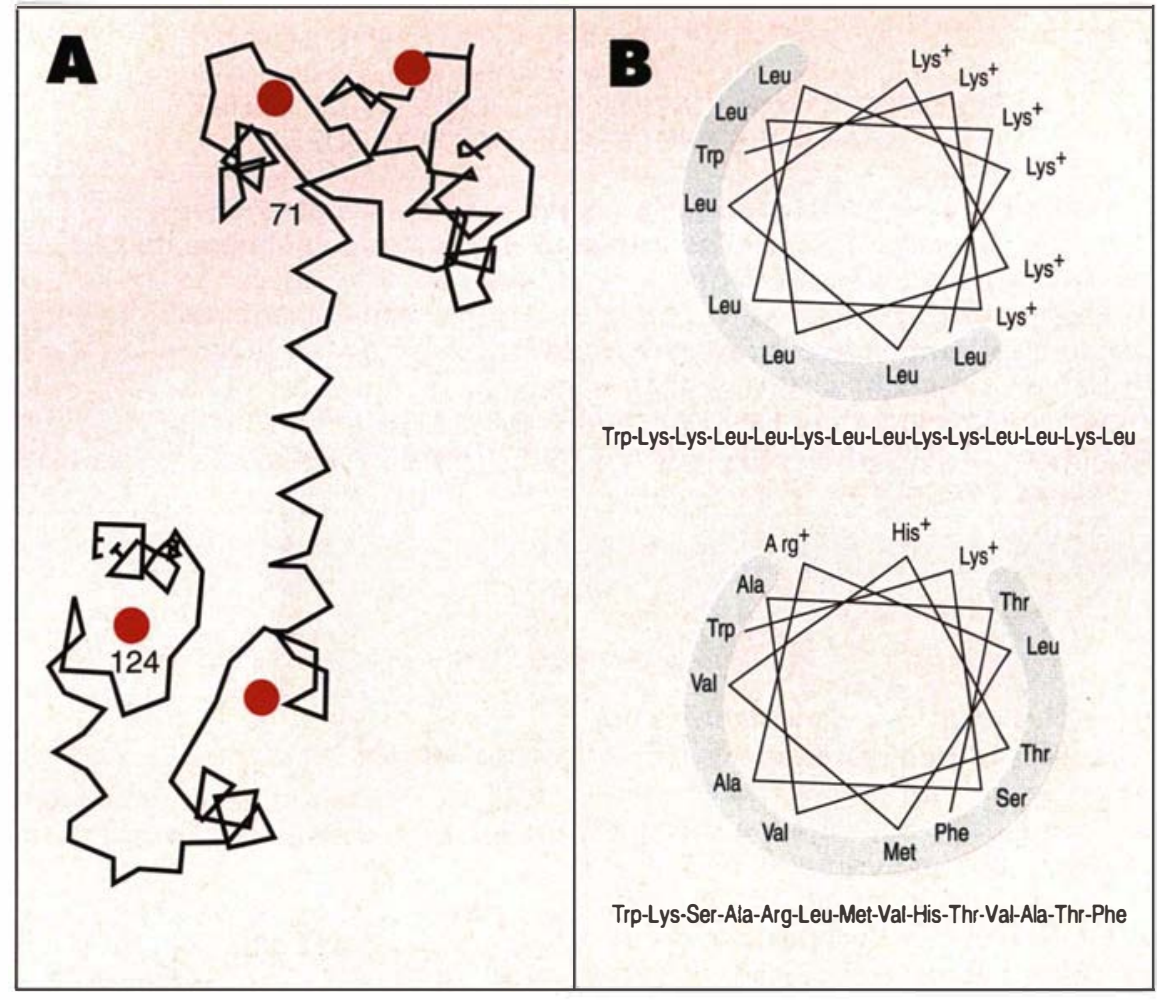

Figure 4. A. Représentation de la chaîne principale de la calmoduline et de la position des 4 sites de fixation du $\mathrm{Ca}^{2+}$ (cercles rouges) [17]. B. Représentation des hélices amphipathiques correspondant (1) au peptide modèle [17] (2) au domaine de fixation de la spectrine à la calmoduline [17].

lum endoplasmique est constituée d'acides aminés basiques, suivie d'une séquence ininterrompue d'acides aminés hydrophobes. Le peptide signal de la mitochondrie et du chloroplaste se caractérise par l'existence de courtes régions hydrophobes séparées par des résidus chargés positivement [16]. Ce type de distribution conduit à une structure hélicoïdale amphiphile composée d'une face essentiellement hydrophile constituée d'acides aminés chargés positivement et d'une face hydrophobe constituée d'acides aminés hydrophobes. Il reste à comprendre comment plusieurs de ces peptides interagissent avec la phase lipidique ou un récepteur spécifique. Dans l'hypothèse d'une interaction avec un récepteur spécifique, il conviendrait peut-être de rechercher dans ce récepteur des motifs amphipathiques complémentaires de ceux que nous venons de décrire. Cette étape de reconnaissance devra cependant être suivie d'un processus beau$\mathrm{m} / \mathrm{s} n^{\circ} 2$, vol. 11, féurier 95 quences très différentes adoptent une structure hélicoïdale amphiphile dans laquelle les acides aminés chargés positivement se rassemblent sur une face de l'hélice. De Grado a synthétisé un peptide modèle intégrant les caractéristiques d'amphiphilicité et d'affinité des peptides s'associant à la calmoduline (figure 4B). Les résidus 1 et 11 ont été remplacés par des résidus phénylalanine portant des groupements capables de se lier de façon covalente aux molécules de leur environnement après photoactivation. Seuls les acides aminés Met124 et Met71, appartenant aux domaines de la calmoduline capables de fixer spécifiquement l'ion $\mathrm{Ca}^{2+}$, sont marqués (figure 4A). La structure cristallographique de complexes calmoduline-peptide confirment que c'est la flexibilité du domaine central de la calmoduline qui permet au peptide de s'associer à 2 domaines différents de la calmoduline [18-20]. Ces données, aussi intéressantes qu'elles soient, ne peuvent néanmoins être extrapolées à la protéine entière qu'avec beaucoup de prudence.

\section{Conclusions et perspectives}

Les exemples que nous venons de décrire font apparaître un dénominateur commun, l'existence d'un motif amphiphile. Dans le cas d'une reconnaissance de constituants membranaires, il n'est pas surprenant que la répartition des domaines hydrophobes et hydrophiles d'un peptide ou d'une protéine détermine son mode d'association à la membrane. La stabilité de la membrane biologique dépend en eff et de l'assemblage harmonieux de motifs amphiphiles. Ainsi, c'est de l'importance relative des domaines hydrophobes (chaînes aliphatiques) et hydrophiles (têtes polaires) que dépend un mode d'organisation en bicouches, en micelles ou en micelles inverses. L'orientation d'un domaine protéique à l'interface lipide-eau sera déterminée davantage par l'importance et la distribution des domaines hydrophobes et hydrophiles que par la nature des acides aminés. Toutefois, l'exemple des séquences signal suggère que cette complémentarité entre domaines hydrophobes et hy- 


\begin{tabular}{|} 
Tableau I \\
EXEMPLES D'IMPLICATION DES HÉLICES AMPHIPHILES \\
DANS LES PROCESSUS BIOCHIMIQUES \\
\hline - Hélices amphiphiles associées aux apolipoprotéines plasmiques. \\
- Hélices amphiphiles dans les protéines du surfactant pulmonaire. \\
- Hélices amphiphiles et peptides cytotoxiques. \\
- Structure amphiphile du peptide signal. \\
- Hélices amphiphiles et neuropeptides. \\
- Activation des protéines G : rôle des hélices amphiphiles. \\
- Rôle de I'hélice amphiphile dans la reconnaissance par les cellules T. \\
- Interaction protéine-DNA: rôle des hélices amphiphiles.
\end{tabular}

D'après [21].

\section{RÉFÉRENCES}

18. Persechini A, Kretsinger RH. The central helix of calmodulin function as a flexible tether. J Biol Chem $1988 ; 263$ : 12175-8.

19. Ikura M, Clore MC, Gronenborn AM, Zhu G, Klee CB, Bax A. Solution structure of a calmodulin - target peptide complex by multidimensional NMR. Science 1992 ; $256: 632-8$.

20. Meador WE, Means AR, Quiocho FA. Target enzyme recognition by calmoduline: 2.4. A structure of a calmoduline-peptide complex. Science 1992 ; 257 : 1251-5.

21. Epand RM. The amphipathic helix. Boca Raton : CRC, 1993.

22. Segrest JP, De Loof $H$, Dohlman JG, Brouillette CG, Anantharamaiah GM. Amphipathic helix motif : classes and properties. Proteins : Structure, Function and Genetics $1990 ; 8$ : 103-17.

23. Challou N, Goormaghtigh E, Cabiaux V, Conrath K, Ruysschaert JM. Sequence and structure of the membrane-associated peptide of glycophorin A. Biochemistry 1994; 33 : $6902-10$.

24. Sonveaux N, Conrath K, Capiau C, Brasseur R, Goormaghtigh E, Ruysschaert JM. The topology of the $S$ protein in the yeastderived hepatitis $\mathrm{B}$ surface antigen particles. J Biol Chem 1994 ; 269 : 2563745.

25. Goormaghtigh E, Vigneron L, Scarborough Gene A, Ruysschaert JM. Tertiary conformational changes of the Neurospora crassa plasma membrane $\mathrm{H}^{+}$-ATPase monitored by hydrogen/deuterium exchange kinetics : a Fourier transform infrared spectroscopy approach. J Biol Chem $1994 ; 269$ : $27409-13$ drophiles concerne également les interactions protéine-protéine.

Une revue récente publiée par R. Epand [21] fait apparaître que l'hélice amphipathique est associée à des activités biologiques très diverses (Tableau I) et cette liste est loin d'être complète. Rien ne permet cependant, pour l'instant, de dire que dans ces différentes situations, le motif amphipathique participe suivant un mécanisme similaire à celui que nous avons décrit. Une compréhension plus moléculaire passe par une approche pluridisciplinaire regroupant les outils de l'analyse conformationnelle et de la mutagenèse dirigée. C'est un préliminaire obligé à toute tentative d'associer à une activité biologique spécifique [22] un motif amphipathique particulier.

Dans le cas de processus membranaires, les analyses structurales de protéines membranaires par cristallographie et résonance magnétique nucléaire se heurtent pour l'instant à des difficultés importantes liées notamment au fait que ces techniques sont délicates à utiliser dans un environnement lipidique. Des techniques spectroscopiques infrarouges se sont avérées mieux adaptées à cet environnement ; elles apportent des informations sur la structure, l'orientation [23, 24] et les modifications de structure tertiaire d'une protéine dans un environnement membranaire [25]

\section{Summary}

Role of the amphipathic helix motif in the recognition process

Ligand-receptor, substrate-enzyme recognition are highly specific processes and minor changes in the ligand or substrate sequence modify drastically this recognition. Several biochemical mechanisms cannot be explained satisfactorily in terms of such a specificity. Calmodulin modulates the activities of a large number of proteins, including protein kinases, phosphodiesterases, calcium pumps. Apolipoproteins with variable sequences have similar biological activities. The number of signal peptides is much more important that the number of membrane targets. A feature common to all the sequences participating to these recognition processes is that they adopt an amphiphilic-helical structure. It is likely that interaction between hydrophilic and hydrophobic domains is responsible for the broad specificity. The distribution of the hydrophobic and hydrophilic domains rather than the exact amino sequence would determine the binding to the target.

\section{Remerciements}

Les travaux du laboratoire dans ce domaine ont bénéficié du concours de E. Goormaghtigh, R. Brasseur, V. Cabiaux, M. Vandenbranden, L. Lins. 